Proteomics applications in Caenorhabditis elegans research

Peer-reviewed author version

Husson, Steven J.; Moyson, Sofie; VALKENBORG, Dirk; Baggerman, Geert \& Mertens, Inge (2015) Proteomics applications in Caenorhabditis elegans research.

In: BIOCHEMICAL AND BIOPHYSICAL RESEARCH COMMUNICATIONS, 468(4), p. 519-524.

DOI: $10.1016 / j . b b r c .2015 .11 .026$

Handle: http://hdl.handle.net/1942/22787 


\section{Proteomics applications in C. elegans research}

Steven J. Husson ${ }^{\mathrm{a}, \mathrm{b}}$, Sofie Moyson ${ }^{\mathrm{a}}$, Dirk Valkenborg ${ }^{\mathrm{b}, \mathrm{c}, \mathrm{d}}$, Geert Baggerman ${ }^{\mathrm{b}, \mathrm{c}}$, Inge Mertens ${ }^{\mathrm{b}, \mathrm{c}}$

${ }^{a}$ Systemic Physiological \& Ecotoxicological Research (SPHERE), Department of Biology, University of Antwerp, Groenenborgerlaan 171, B-2020 Antwerp, Belgium

${ }^{\mathrm{b}}$ Center For Proteomics (CFP-CEPROMA), University of Antwerp, Groenenborgerlaan 171, B-2020 Antwerp, Belgium

${ }^{\mathrm{c}}$ Flemish Institute for Technological Research (VITO), Boeretang 200, B-2400 Mol, Belgium

${ }^{\mathrm{d}}$ Interuniversity Institute for Biostatistics and statistical Bioinformatics, UHasselt, Agoralaan 1, B-3590 Diepenbeek, Belgium

Correspondence should be addressed to: Steven J. Husson, steven.husson@ uantwerpen.be

Running title: Worm Proteomics

List of abbreviations: PD: Parkinson's disease; SILAC: Stable isotope labeling with amino acids in cell culture; iTRAQ: Isobaric tag for relative and absolute quantitation; ICAT: Isotope coded affinity tag; PTM: Post-translational modification.

No conflicts of interest are declared 


\begin{abstract}
The free-living nematode Caenorhabditis elegans is one of the most studied models in a wide variety of research fields with applications in agro- or pharmaceutical industries. It has been used for the development of new anthelminthic drugs and was proven to yield key insights in neurodegenerative diseases and metabolic syndromes. Due to its suitability for highthroughput genetic screens, efficiency for RNA interference approaches and the availability of thousands of mutants, most studies were carried out at the genetic level. However, determining the cellular function of each gene product remains an unfinished goal in this postgenomic era. A systems biology approach focusing on the actual gene products (i.e. proteins) can help unraveling this puzzle. A fundamental pillar in this research is mass spectrometrybased proteomics. We here provide an in-depth overview of proteomics-related studies in $C$. elegans research, with special emphasis on the methodologies and biological applications.
\end{abstract}

Keywords: Caenorhabditis elegans; peptidomics; proteomics; systems biology; biological applications 


\section{Introduction: $C$. elegans as a model system}

The soil nematode Caenorhabditis elegans affords many advantages for fundamental research in a wide variety of disciplines. It has a defined cell lineage, a completed genome sequence, a life cycle of about three days, a short life span of only 2-3 weeks and it contains 959 somatic cells including 302 neurons for which the connectivity map has been determined [1]. Also, it is easy and inexpensive to maintain in the lab and it has a very small body size so that in vivo assays can be conducted in 96-well plates. Moreover, human homologs have been identified for at least $50 \%$ of the more than 19.000 C. elegans genes [2], while this soil nematode can also be considered as a systems model for parasitic nematodes as to aid development of novel anthelmintic drugs. These advantages, together with the wealth of genetic methods, made $C$. elegans shift from a mere developmental model towards a model to study human-related diseases and to perform drug target screens. C. elegans is used in both mechanistic studies and high-throughput screening approaches in the areas of neurotoxicology, genetic toxicology and environmental toxicology [3]. These high-throughput screenings include genome-wide screenings for molecular targets of toxicity and rapid toxicity assessment for new chemicals. Compared to the mammalian models, more compounds can be tested while reducing expense and sacrificing fewer mammals.

In general, $C$. elegans research mainly focused on genomic-based approaches, making use of forward or reversed genetic screens for which this model system is ideally suited. However, proteins that are in fact responsible for the biological action were largely ignored. Several technical innovations made in the last decade to allow comprehensive analysis of the proteome, peptidome and interactome, enormously contributed to the completion of the systems biology puzzle. In addition, C. elegans is often used as a reproducible source to obtain complex protein samples to be used to develop, optimize or validate technological hardware and software innovations in the field of proteomics. We here provide an overview 
of technological innovations for which $C$. elegans has been used as biological starting material, and further discuss fundamental biological insights obtained by using proteomics approaches.

\section{Technological innovations}

\subsection{Instrumentation and software}

Most studies focus on a spatiotemporal selection of the proteome that is important in a given biological context. The protein spectrum of the subjected proteome is then further limited by the performance of the analytical and technological tools used to assess quantitative and qualitative data. During the last years, there was a considerable progress in high-throughput proteomics analysis and data integration due to the continuous technological improvements on instrumentation. For example, the introduction of the dual-pressure linear ion trap mass spectrometer (Thermo Fisher Scientific LTQ Velos) features increased sensitivity and faster cycle times and therefore resulted in a substantial increase in the detection and identification of both proteins and unique peptides from the complex C. elegans proteome [4]. In a 60 minutes LC separation of $1 \mu \mathrm{g}$ of $C$. elegans digest, more than 1500 proteins could be identified using the LTQ Velos, compared to +/- 800 proteins on an LTQ XL. C. elegans was also used as source material to develop and validate a novel plug and play LC-MS source [5], to evaluate of a front-end higher energy collision-induced dissociation (fHCD) on a benchtop dual-pressure linear ion trap instrument [6], to introduce a user-friendly web interface for predicting gene functions (GeneMANIA) [7] and to launch a web server (SteinerNet) for integrating "omics" data [8]. 


\subsection{Quantitative proteomics}

\subsubsection{Metabolic labeling}

For quantitative proteomics studies of cell cultures, the so-called "stable-isotope labeling by amino acids in cell culture (SILAC)" has been introduced by the Mann group in 2002 [9]. In line with this idea, Krijgsveld and coworkers from the Heck lab labeled Drosophila melanogaster and C. elegans with ${ }^{15} \mathrm{~N}$ in 2003 [10], by feeding them on ${ }^{15} \mathrm{~N}$-labeled yeast or Escherichia coli, respectively. This labeling strategy was thus achieved in two steps: first, $E$. coli was grown in medium enriched in ${ }^{15} \mathrm{~N}$ to achieve $>98 \%$ labeling. Next, labeled $E$. coli was spread over agar plates lacking nutrients and any other potential nitrogen source. A detailed methodology was recently described, in addition to bioinformatics methods for the analysis of generated MS spectra [11]. In 2011, two papers in the same issue of Nature Methods described the use of heavy isotope-labeled lysine and arginine to label Escherichia coli, to be used as a food source for the nematodes [12;13]. Protein perturbations as a result of RNAi-mediated knock down of the nuclear hormone receptor 49 was investigated by the Faergeman group [12], while the heat-shock response was studied in the Lamond lab [13].

\subsubsection{Chemical labeling (in vitro)}

Obviously, classical chemical labeling approaches like iTRAQ, ICAT or TMT (which are not discussed here) can be used to label $C$. elegans peptides in vitro. Fluorogenic derivatization (FD) of protein mixtures is an alternative labeling method that has been used in C. elegans. First, chemical labeling is achieved by 7-chloro-4-(dimethylaminoethylaminosulfonyl)-2,1,3benzoxadiazole (DAABD-Cl), followed by HPLC isolation, enzymatic digestion and identification of the derivatized proteins by HPLC-ESI-MS/MS [14]. This method was used for the identification of negative markers at early stages of Parkinson's disease [15]. 


\subsubsection{Label-free methods for relative quantification}

The group of Yates improved the data acquisition strategy to a data-independent method that permits quantitative analysis directly from tandem mass spectra [16]. The method is based on the sequential isolation and fragmentation of relative large $(10 \mathrm{~m} / \mathrm{z})$ precursor windows within the ion trap until a desired mass range has been covered. Validation of the method was performed by using different developmental stages of C. elegans. They also used this method to identify insulin signaling targets in C. elegans [17]. Of 1685 identified proteins, 86 were over- or under expressed in long-lived daf-2 mutants that lack the sole homolog of the vertebrate insulin-receptor or IGF-1 receptor, compared to wild-type worms.

\section{Biological applications}

Classical, non-LC-MS based proteomics studies in nematodes using 2D gel electrophoresis, Western Blots, immunoprecipitation, etc. led, for example, to the identification of new conserved components of the kinetochore [18;19], of the RNAi machinery and of the microRNA pathway [20]. Classical approaches were also used to identify all phosphorylcholine substituted proteins, called the PCome [21]. However, most other qualitative proteome studies in C. elegans are LC-MS based, as illustrated below.

\subsection{Shotgun proteomics to aid species comparisons and gene annotations}

The group of Merrihew used shotgun proteomics to identify 6779 proteins in C. elegans [22]. One year later, Schrimpf et al. made an inventory of the expressed proteome yielding 10977 
different proteins, which is more than half of all predicted C. elegans proteins [23]. It allowed them to confirm and extent genome annotations. In addition, they made a comparative functional analysis of the C. elegans and Drosophila proteomes.

\subsection{Subproteomes}

In some cases, a subset of proteins is defined by their intracellular location. The group of Yang published the proteomic analysis of C. elegans mitochondria [24] using a shotgun approach to identify 1117 proteins. In order to search for diagnostic fingerprints of mitochondrial diseases, differential proteomics analysis of mitochondria from mutants of the respiratory chain yielded 630 proteins in wild type N2, 525 in $c l k-1$ (encoding for COQ7, which catalyzes the penultimate step of coenzyme Q synthesis), 487 in gas-1 (orthologue of NDUFS2, a 49kDa subunit of complex I of the mitochondrial respiratory chain), and 423 in mev-1 (orthologue of SDHC, a subunit of complex II) [25]. Cross-correlation with metabolomics profiling data and expression studies showed maintained patterns, which might aid further understanding of mitochondrial diseases.

Lipid droplets (LDs) are neutral lipid storage organelles that are highly conserved. Overstorage of neutral lipids in LDs can cause many metabolic syndromes. Purification and proteomic analysis of C. elegans LDs in the Liu group yielded 306 proteins, further facilitating functional and mechanistic studies of LDs [26]. Lipid rafts are microdomains (islands) of the phospholipid bilayer and most proteomics studies to reveal lipid raft composition have been carried out using mammalian cell lines or single cell organisms. Using geLC-MS/MS, a total of 44 proteins could be identified from the lipid raft fraction of $C$. elegans by the Keen lab and 21 proteins were predicted to contain a GPI anchor [27]. Conserved nematode-specific intestinal proteins may represent promising targets for the 
development of anthelmintic drugs. The analysis of membrane associated proteins from parasitic nematodes was recently optimized using ivermectin resistant and ivermectin susceptible isolates of C. elegans and Haemonchus contortus [28] in the Hamilton lab. Recently, the Tirrell lab achieved spatiotemporal selectivity in the labeling of proteins as to yield cell-specific subproteomes, which was exemplified by profiling proteins expressed in the pharyngeal muscle [29].

\subsection{Reproduction and Development}

The extremely consistent developmental cycle of 3-4 days from fertilized egg to adult through four larval stages (L1 to L4) is well studied on the morphological level, as the complete cell lineage of the animal has been recorded. Under unfavorable conditions like starvation, an alternative "dauer" or diapause stage can be reached. This alternative, hibernated life form is adapted to harsh environments and remains viable for several months. However, when encountering a food source, they can re-enter the L4 stage and develop to reproductive adults. About ten years ago, the Glocker group used a gel-based approach to reveal dynamic cytoskeletal processes during ontogenesis by analyzing protein maps of different developmental stages [30]; effects of cultivation temperatures were also analyzed [31]. The Tsugita lab categorized proteins observed in different developmental stages in subgroups according to their stage-specific abundances [32]. A similar study quantifying proteomes at different time points during ontogenesis using $\mathrm{N}^{15}$-labeling and LC-MS/MS analysis confirmed observed changes for selected proteins [33]. Recently, a SILAC approach using

${ }^{13} \mathrm{C}_{6}$-Lys labeled $E$. coli as food source for the nematodes revealed that the rate of appearance of newly synthesized proteins over the adult life span was high during the first 5 days of adulthood, slowed down until the $11^{\text {th }}$ day to increase again afterwards [34]. The McGhee lab 
specifically focused on the subproteome from oocytes that were isolated from a temperaturesensitive fertilization-defective fer-1 mutant [35]. When comparing this dataset with a previously determined oocyte transcriptome, striking differences could be noted, illustrating the need for biochemical analysis to complement genome and transcriptome-based omics data in a systems biology approach. The oocyte proteome seemed to be enriched for proteins that are presumably required immediately upon fertilization, whereas transcriptome data pointed to enriched molecules for later stages in embryogenesis [35]. In order to investigate molecular processes underlying oogenesis and spermatogenesis separately, the Krijgsveld group employed their initially reported $\mathrm{N}^{15}$ metabolic labeling strategy to compare two mutant lines that develop only male-like $(f e m-3(q 20))$ or female-like $(f e m-1(h c 17))$ animals [36]. From 1040 identified proteins, $\sim 200$ and $\sim 170$ were upregulated in female-like and male-like animals, respectively; some of which could be clearly linked to spermatogenesis or oogenesis. For example, CAR-1 CGH-1 and CEY-2/3 are strikingly more abundant in female-like worms. These proteins associate in $\mathrm{P}$ granules that segregate asymmetrically into precursor cells yielding the germline. As also indicated above, comparing this dataset with a previously published microarray study [37] showed poor correlations. Effects of ionizing radiation on the germlines were investigated in the group of Thiede. Using stringent criteria, 48 proteins were significantly up or down regulated, most of which are (indirectly) involved in translational control. Interestingly, the before-mentioned CAR-1/CGH-1/CEY-3 complex was found to act as a negative regulator of DNA damage-induced apoptosis [38]. A 2D-DIGE study performed by the Shim lab revealed that IFE-1, a C. elegans homolog of eIF4E (which is the mRNA 5' cap-binding component of the translation initiation complex eIF4F), regulates expression of sperm-specific proteins [39]. The same group also reported that cholesterol-responsive metabolic proteins are required for larval development in C. elegans [40]. The intestinal peptide transporter PEPT-1 seems to be important for development, growth, reproduction and 
stress tolerance as revealed by the phenotype of respective mutants. A role of PEPT-1 in cellular amino acid homeostasis by affecting the TOR complex (target of rapamycine, the cellular sensor for free amino acids) and the XBP-1 pathway (the major mediator of the unfolded protein response) was found by quantitative proteome analysis combined with transcriptome profiling in the Spanier lab [41].

\subsection{Aging and longevity}

C. elegans is a well-respected animal model to elucidate fundamental aspects of aging. The insulin-like growth factor-1 (IGF-1) signaling pathway is a key regulator of aging in the worm, and similar findings are documented for mice and flies. Reduced IGF-1 signaling via the DAF-2 receptor results in doubling of lifespan [42] and daf-2 mutants, together with dauer animals, are considered as important models to study longevity. Several differential proteomics studies were performed to unravel underlying mechanisms of aging. The Yates group aimed to identify (regulators of) insulin signaling targets by comparing daf-2 animals with wild type counterparts using $\mathrm{N}^{15}$-based proteomics analysis that yielded 86 differentially present proteins out of 1685 proteins identified. Validation of target molecules was performed by selected reaction monitoring (SRM) and Western blotting, followed by characterization of a subset of target molecules by RNAi [43]. When comparing the protein content of L3 larvae with dauers, a metabolic shift to anaerobic fermentative pathways was observed [44]. A few years later, the Hamilton group reported shared detoxification systems in dauer larvae and daf-2 mutants and supported a diversion of energy consumption away from anabolic processes in these two aging models [45]. Recently, a multidisciplinary approach involving quantitative proteomics, immunochemistry, metabolic quantification and life span analysis pointed to an enhanced energy metabolism contributing extended life span of calorie- 
restricted (CR) animals, for which they used eat-2 mutants [46]. The Braeckman group found that general translation mechanisms are inhibited by reduced IGF-1 signaling and dietary restriction, while the presence of many structural muscle components are preserved in longlived worms [47]. Furthermore, reduced protein metabolism [48] and faster transition to reducing conditions [49] were recently found in IGF-1-mediated long-living animals. To study the contribution of protein aggregation in aging and to identify modifiers of longevity, the SDS-insoluble protein fraction of young and aged animals were compared, followed by knockdown of genes encoding insoluble proteins observed in aged nematodes and subsequent testing in a lifespan assay [50]. The underlying mechanisms of tyrosol (one of the main phenols in olives) on increased resistance to thermal and oxidative stress and extension of lifespan in nematodes was recently assayed using a differential proteomics approach [51]. Similarly, proteins related to heat stress tolerance, energy metabolism and muscle structure seemed to be upregulated upon treatment of worms with Tasco, a commercial product originating from the alga Ascophyllum nodosum that was found to induce enhanced thermal stress tolerance and extended life span [52]. Finally, the lifespan-extending properties of metformin, the antiglycemic drug to treat type II diabetes, were found to act through mitohormesis via the peroxiredoxin PRDX-2 [53]. An overview of proteomics approaches to study oxidative stress in aging can be found in [54].

\subsection{Innate immunity}

Only few comprehensive proteomics studies were performed to understand underlying mechanisms of innate immunity to selected bacterial or fungal infections of C. elegans. Two gel-based approaches were performed in the Schoofs lab to study effects of Aeromonas hydrophila [55] and Staphylococcus aureus [56]. A similar 2D-DIGE approach revealed the 
significance of unfolded protein response (UPR) pathway during bacterial infection with Vibrio alginolyticus in the Balamurugan group [57]. In addition, some of the upregulated proteins were involved in stress-response (PDI-2, HSP-6), immune-response (protein kinase 18, GST-8) and energy-production (ATP-2) while proteins involved in structural maintenance (IFB-2) and lipid metabolism (SODH-1) were downregulated. Immune response pathways upon infection with the E. coli strain LF82, which is endogenous to patients suffering from Crohn's disease or inflammatory bowel disease, was investigated in the Kallipolitis lab to identify ferritin (FTN-2) to be essential for a full protective response [58]. The Ewbank lab

was able to characterize HSP-3 as the worm orthologue of mammalian BiP/GRP78 (a chaperone protein in the ER) to control antimicrobial peptide (AMP) expression in the epidermis upon infection to Drechmeria coniospora [59]. Recently, data from a differential proteomics approach using 2D-LC MS and iTRAQ labeling was combined with transcriptomic analysis by RNA-Seq in the Schulenburg group to reveal the involvement of C-type lectin domain-containing proteins and AMP-activated protein kinases in immune defense mechanisms against Bacillus thuringiensis [60]. A similar iTRAQ-based 2D-LC MS setup also revealed transthyretin-like proteins, lysozymes and galectins next to the C-type lectins to be differentially regulated upon exposure with the same pathogen, $B$. thuringiensis, as found in the Tholey group [61]. An overview of transcriptomics and proteomics approaches to study $C$. elegans innate immunity and host-pathogen interactions are described in [62].

\subsection{Glycoproteins and phosphoproteomics}

Other qualitative studies focus on a subset of proteins, like hydrophobic glycoproteins and phosphoproteins. Posttranslational modifications (PTMs) in general are of particular interest 
because they can serve as regulatory switches. By doing so, they control several biological functions. Of the $\sim 200$ different known PTMs, glycosylation and phosphorylation are the most common PTMs in eukaryotes. In 2005, the group of Mahuran already made a first inventory of hydrophobic glycoproteins [63]. They developed a proteomics method that was able to identify 117 hydrophobic N-glycosylated proteins in C. elegans by analyzing 195 glycopeptides containing $199 \mathrm{~N}$-linked oligosaccharides. Most of the proteins identified were involved in cell adhesion, metabolism or the transport of small molecules. The N-glycans that are predominantly present in $C$. elegans are oligomannose and paucimannose, which are usually not present in vertebrates. Both $\mathrm{N}$-glycans bind strongly to the lectin Concanavalin A (Con A), so that the capture of C. elegans $\mathrm{N}$-glycans by Con A is predicted to be well over $90 \%$. The ability to use Con A to purify glycoproteins or glycopeptides was already described by Kaji et al. in 2003 [64]. The Paik lab evaluated O-GlcNAc modifications in dauer worms [65]. These PTMs are known to interact competitively or synergistically with protein phosphorylation and are of key importance to cells for their timely response to extracellular stimuli.

The Mann group characterized the C. elegans phosphoproteome to detect 6780 phosphorylation sites on 2373 proteins; results were integrated together with a new prediction application in PHOSIDA [66].

\subsection{Peptidomics}

Using a variety of LC-MS-based approaches, endogenously occurring (on-tryptic) (neuro)peptides could be identified from C. elegans [67], C. briggsae [68] and Ascaris suum [69], as recently reviewed [70]. For a robust biochemical characterization of presumed processing enzymes, differential peptidomics approaches were undertaken to monitor the 
peptide content of respective mutants by off line HPLC - MALDI TOF MS. This way, the Kex-2 like proprotein convertase 2 (KPC-2, also named EGL-3) [71], its chaperone protein 7B2 (encoded by sbt-1) [72] and the carboxypeptidase E (EGL-21) [73] could be characterized.

\subsection{Interactomics}

The first map of the interactome network of C. elegans was published in 2004 by the research group of Vidal [74]. Starting with a selected set of 3024 worm predicted proteins, they obtained over 4000 interactions. This data was combined with literature, interologs and scaffold data sets in Worm Interactome version 5, containing 5534 interactions. The interactions connected $15 \%$ of the C. elegans proteome. Later-on, the same group improved the protein-protein interactions data by testing a matrix of $10.000 \times 10.000$ proteins using HTY2H [75]. They also developed new standards to deliver high quality data sets. These involve a highly stringent, high throughput $\mathrm{Y} 2 \mathrm{H}$ assay, strict methods for filtering and updating existing data sets, independent measurement of technical quality and evaluation of biological relevance. The Sternberg lab integrated available interactomics data, gene expression information, phenotype datasets and functional annotations in a statistical model to predict genetic interactions. The resulting 18.183 interactions not only include protein-protein interactions, but also protein-gene and gene-gene interactions and they provide a framework for systems biology-level understanding of gene functions [76].

\section{Wormbase}


In the post-genomic era, the main goal of all technological innovations is to define the role and function of each gene product in the complex cellular context. This not only means that the function of a single gene product has to be revealed, but also that the signal transduction pathway of the protein of interest and all its interactions and modifications have to be characterized. This is an enormous challenge and to reach that goal, an overload of information has to be included in this systems biology puzzle. Not only genes and proteins, but also phenotypes resulting from RNAi screens or from genomic mutants, localization studies, etc., are indispensable for the "worm community". In a first attempt to organize all available information, a freely accessible database (http://www.wormbase.org) was developed in 2001 [77]. Since then, this repository of all data related to C. elegans biology has been thoroughly restructured for stronger performance and included data has been significantly expanded. In 2012, a drastic makeover of the website was performed and even more functionalities were made available. As such, it should be considered as a complete knowledge-database fueling a systems biology understanding of the nematode C. elegans and beyond.

\section{References}

[1] J.G. White, E. Southgate, J.N. Thomson, S. Brenner, The structure of the nervous system of the nematode Caenorhabditis elegans. Philos.Trans.R.Soc.Lond.B.Biol.Sci. 314 (1986) 1-340.

[2] The C.elegans sequencing consortium, Genome sequence of the nematode C. elegans: a platform for investigating biology. Science 282 (1998) 2012-2018.

[3] M.C. Leung, P.L. Williams, A. Benedetto, C. Au, K.J. Helmcke, M. Aschner, J.N. Meyer, Caenorhabditis elegans: an emerging model in biomedical and environmental toxicology. Toxicol.Sci. 106 (2008) 5-28. 
[4] M.R. Hoopmann, G.E. Merrihew, P.D. von Haller, M.J. Maccoss, Post analysis data acquisition for the iterative MS/MS sampling of proteomics mixtures. J.Proteome.Res. 8 (2009) 1870-1875.

[5] M.S. Bereman, E.J. Hsieh, T.N. Corso, C.K. Van Pelt, M.J. Maccoss, Development and characterization of a novel plug and play liquid chromatography-mass spectrometry (LC-MS) source that automates connections between the capillary trap, column, and emitter. Mol.Cell Proteomics. 12 (2013) 1701-1708.

[6] M.S. Bereman, J.D. Canterbury, J.D. Egertson, J. Horner, P.M. Remes, J. Schwartz, V. Zabrouskov, M.J. Maccoss, Evaluation of front-end higher energy collision-induced dissociation on a benchtop dual-pressure linear ion trap mass spectrometer for shotgun proteomics. Anal.Chem. 84 (2012) 1533-1539.

[7] D. Warde-Farley, S.L. Donaldson, O. Comes, K. Zuberi, R. Badrawi, P. Chao, M. Franz, C. Grouios, F. Kazi, C.T. Lopes, A. Maitland, S. Mostafavi, J. Montojo, Q. Shao, G. Wright, G.D. Bader, Q. Morris, The GeneMANIA prediction server: biological network integration for gene prioritization and predicting gene function. Nucleic Acids Res. 38 (2010) W214-W220.

[8] N. Tuncbag, S. McCallum, S.S. Huang, E. Fraenkel, SteinerNet: a web server for integrating 'omic' data to discover hidden components of response pathways. Nucleic Acids Res. 40 (2012) W505-W509.

[9] S.E. Ong, B. Blagoev, I. Kratchmarova, D.B. Kristensen, H. Steen, A. Pandey, M. Mann, Stable isotope labeling by amino acids in cell culture, SILAC, as a simple and accurate approach to expression proteomics. Mol.Cell Proteomics. 1 (2002) 376-386.

[10] J. Krijgsveld, R.F. Ketting, T. Mahmoudi, J. Johansen, M. Artal-Sanz, C.P. Verrijzer, R.H. Plasterk, A.J. Heck, Metabolic labeling of C. elegans and D. melanogaster for quantitative proteomics. Nat.Biotechnol. 21 (2003) 927-931.

[11] J.W. Gouw, B.B. Tops, J. Krijgsveld, Metabolic labeling of model organisms using heavy nitrogen (15N). Methods Mol.Biol. 753 (2011) 29-42.

[12] J. Fredens, K. Engholm-Keller, A. Giessing, D. Pultz, M.R. Larsen, P. Hojrup, J. Moller-Jensen, N.J. Faergeman, Quantitative proteomics by amino acid labeling in $C$. elegans. Nat.Methods 8 (2011) 845-847.

[13] M. Larance, A.P. Bailly, E. Pourkarimi, R.T. Hay, G. Buchanan, S. Coulthurst, D.P. Xirodimas, A. Gartner, A.I. Lamond, Stable-isotope labeling with amino acids in nematodes. Nat.Methods 8 (2011) 849-851.

[14] M. Masuda, C. Toriumi, T. Santa, K. Imai, Fluorogenic derivatization reagents suitable for isolation and identification of cysteine-containing proteins utilizing highperformance liquid chromatography-tandem mass spectrometry. Anal.Chem. 76 (2004) 728-735.

[15] T. Ichibangase, H. Saimaru, N. Takamura, T. Kuwahara, A. Koyama, T. Iwatsubo, K. Imai, Proteomics of Caenorhabditis elegans over-expressing human $\alpha$-synuclein analysed by fluorogenic derivatization-liquid chromatography/tandem mass 
spectrometry: identification of actin and several ribosomal proteins as negative markers at arly Parkinson's disease stages. Biomed.Chromatogr. 22 (2008) 232-234.

[16] J.D. Venable, M.-Q. Dong, J. Wohlschlegel, A. Dillin, J.R. Yates, Automated approach for quantitative analysid of complex peptide mixtures from tandem mass spectra. Nat.Methods 1 (2004) 1-7.

[17] M.Q. Dong, J.D. Venable, N. Au, T. Xu, S.K. Park, D. Cociorva, J.R. Johnson, A. Dillin, J.R. Yates, III, Quantitative mass spectrometry identifies insulin signaling targets in C. elegans. Science 317 (2007) 660-663.

[18] I.M. Cheeseman, S. Niessen, S. Anderson, F. Hyndman, J.R. Yates, III, K. Oegema, A. Desai, A conserved protein network controls assembly of the outer kinetochore and its ability to sustain tension. Genes Dev. 18 (2004) 2255-2268.

[19] I.M. Cheesemann, J.S. Chappie, E.M. Wilson-Kubalek, A. Desai, The conserved KMN network constitues the core microtubule-binding site of the kinotochore. Cell 127 (2006) 983-997.

[20] L. Zhang, L. Ding, T.H. Cheung, M.Q. Dong, J. Chen, A.K. Sewell, X. Liu, J.R. Yates, III, M. Han, Systematic identification of C. elegans miRISC proteins, miRNAs, and mRNA targets by their interactions with GW182 proteins AIN-1 and AIN-2. Mol.Cell 28 (2007) 598-613.

[21] J. Grabitzki, M. Ahrend, H. Schachter, R. Geyer, G. Lochnit, The PCome of Caenorhabditis elegans as a prototypic model system for parasitic nematodes: identification of phosphorylcholine-substituted proteins. Mol.Biochem.Parasitol. 161 (2008) 101-111.

[22] G.E. Merrihew, C. Davis, B. Ewing, G. Williams, L. Kall, B.E. Frewen, W.S. Noble, P. Green, J.H. Thomas, M.J. Maccoss, Use of shotgun proteomics for the identification, confirmation, and correction of C. elegans gene annotations. Genome Res. 18 (2008) 1660-1669.

[23] S.P. Schrimpf, M. Weiss, L. Reiter, C.H. Ahrens, M. Jovanovic, J. Malmstrom, E. Brunner, S. Mohanty, M.J. Lercher, P.E. Hunziker, R. Aebersold, M.C. von, M.O. Hengartner, Comparative functional analysis of the Caenorhabditis elegans and Drosophila melanogaster proteomes. PLoS.Biol. 7 (2009) e48.

[24] Li J., Cai T., Wu P., Cui Z., Chen X., Hou J., Xie Z., Xue P., Shi L., Liu P., Yates J.R., Yang F., Proteomic analysis of mitochondria from Caenorhabditis elegans. Proteomics 9 (2009) 1-15.

[25] P.G. Morgan, R. Higdon, N. Kolker, A.T. Bauman, O. Ilkayeva, C.B. Newgard, E. Kolker, L.M. Steele, M.M. Sedensky, Comparison of proteomic and metabolomic profiles of mutants of the mitochondrial respiratory chain in Caenorhabditis elegans. Mitochondrion. 20 (2015) 95-102.

[26] P. Zhang, H. Na, Z. Liu, S. Zhang, P. Xue, Y. Chen, J. Pu, G. Peng, X. Huang, F. Yang, Z. Xie, T. Xu, P. Xu, G. Ou, S.O. Zhang, P. Liu, Proteomic study and marker protein identification of Caenorhabditis elegans lipid droplets. Mol.Cell Proteomics. 11 (2012) 317-328. 
[27] W. Rao, R.E. Isaac, J.N. Keen, An analysis of the Caenorhabditis elegans lipid raft proteome using geLC-MS/MS. J.Proteomics. 74 (2011) 242-253.

[28] E.H. Hart, P.M. Brophy, M. Prescott, D.J. Bartley, B.T. Wolf, J.V. Hamilton, A new enabling proteomics methodology to investigate membrane associated proteins from parasitic nematodes: case study using ivermectin resistant and ivermectin susceptible isolates of Caenorhabditis elegans and Haemonchus contortus. Vet.Parasitol. 207 (2015) 266-275.

[29] K.P. Yuet, M.K. Doma, J.T. Ngo, M.J. Sweredoski, R.L. Graham, A. Moradian, S. Hess, E.M. Schuman, P.W. Sternberg, D.A. Tirrell, Cell-specific proteomic analysis in Caenorhabditis elegans. Proc.Natl.Acad.Sci.U.S.A 112 (2015) 2705-2710.

[30] A. Madi, S. Mikkat, B. Ringel, H.J. Thiesen, M.O. Glocker, Profiling stage-dependent changes of protein expression in Caenorhabditis elegans by mass spectrometric proteome analysis leads to the identification of stage-specific marker proteins. Electrophoresis 24 (2003) 1809-1817.

[31] A. Madi, S. Mikkat, B. Ringel, M. Ulbrich, H.J. Thiesen, M.O. Glocker, Mass spectrometric proteome analysis for profiling temperature-dependent changes of protein expression in wild-type Caenorhabditis elegans. Proteomics. 3 (2003) 1526-1534.

[32] Y. Tabuse, T. Nabetani, A. Tsugita, Proteomic analysis of protein expression profiles during Caenorhabditis elegans development using two-dimensional difference gel electrophoresis. Proteomics. 5 (2005) 2876-2891.

[33] K.E. Geillinger, K. Kuhlmann, M. Eisenacher, H.E. Meyer, H. Daniel, B. Spanier, Dynamic changes of the Caenorhabditis elegans proteome during ontogenesis assessed by quantitative analysis with $15 \mathrm{~N}$ metabolic labeling. J.Proteome.Res. 11 (2012) 45944604.

[34] K. Vukoti, X. Yu, Q. Sheng, S. Saha, Z. Feng, A.L. Hsu, M. Miyagi, Monitoring Newly Synthesized Proteins over the Adult Life Span of Caenorhabditis elegans. J.Proteome.Res. 14 (2015) 1483-1494.

[35] J.K. Chik, D.C. Schriemer, S.J. Childs, J.D. McGhee, Proteome of the Caenorhabditis elegans oocyte. J.Proteome.Res. 10 (2011) 2300-2305.

[36] B.B. Tops, S. Gauci, A.J. Heck, J. Krijgsveld, Worms from venus and mars: proteomics profiling of sexual differences in Caenorhabditis elegans using in vivo $15 \mathrm{~N}$ isotope labeling. J.Proteome.Res. 9 (2010) 341-351.

[37] V. Reinke, I.S. Gil, S. Ward, K. Kazmer, Genome-wide germline-enriched and sexbiased expression profiles in Caenorhabditis elegans. Development 131 (2004) 311323.

[38] G.G. Tomazella, H. Kassahun, H. Nilsen, B. Thiede, Quantitative proteome analysis reveals RNA processing factors as modulators of ionizing radiation-induced apoptosis in the C. elegans germline. J.Proteome.Res. 11 (2012) 4277-4288.

[39] I. Kawasaki, M.H. Jeong, Y.H. Shim, Regulation of sperm-specific proteins by IFE-1, a germline-specific homolog of eIF4E, in C. elegans. Mol.Cells 31 (2011) 191-197. 
[40] I. Kawasaki, M.H. Jeong, Y.J. Yun, Y.K. Shin, Y.H. Shim, Cholesterol-responsive metabolic proteins are required for larval development in Caenorhabditis elegans. Mol.Cells 36 (2013) 410-416.

[41] K.E. Geillinger, K. Kuhlmann, M. Eisenacher, P. Giesbertz, H.E. Meyer, H. Daniel, B. Spanier, Intestinal amino acid availability via PEPT-1 affects TORC1/2 signaling and the unfolded protein response. J.Proteome.Res. 13 (2014) 3685-3692.

[42] K.D. Kimura, H.A. Tissenbaum, Y. Liu, G. Ruvkun, daf-2, an insulin receptor-like gene that regulates longevity and diapause in Caenorhabditis elegans. Science 277 (1997) 942-946.

[43] M.Q. Dong, J.D. Venable, N. Au, T. Xu, S.K. Park, D. Cociorva, J.R. Johnson, A. Dillin, J.R. Yates, III, Quantitative mass spectrometry identifies insulin signaling targets in C. elegans. Science 317 (2007) 660-663.

[44] A. Madi, S. Mikkat, C. Koy, B. Ringel, H.J. Thiesen, M.O. Glocker, Mass spectrometric proteome analysis suggests anaerobic shift in metabolism of Dauer larvae of Caenorhabditis elegans. Biochim.Biophys.Acta 1784 (2008) 1763-1770.

[45] L.M. Jones, K. Staffa, S. Perally, E.J. LaCourse, P.M. Brophy, J.V. Hamilton, Proteomic analyses of Caenorhabditis elegans dauer larvae and long-lived daf-2 mutants implicates a shared detoxification system in longevity assurance. $\mathrm{J}$ Proteome.Res. 9 (2010) 2871-2881.

[46] Y. Yuan, C.S. Kadiyala, T.T. Ching, P. Hakimi, S. Saha, H. Xu, C. Yuan, V. Mullangi, L. Wang, E. Fivenson, R.W. Hanson, R. Ewing, A.L. Hsu, M. Miyagi, Z. Feng, Enhanced energy metabolism contributes to the extended life span of calorie-restricted Caenorhabditis elegans. J.Biol.Chem. 287 (2012) 31414-31426.

[47] G. Depuydt, F. Xie, V.A. Petyuk, N. Shanmugam, A. Smolders, I. Dhondt, H.M. Brewer, D.G. Camp, R.D. Smith, B.P. Braeckman, Reduced insulin/IGF-1 signaling and dietary restriction inhibit translation but preserve muscle mass in Caenorhabditis elegans. Mol.Cell Proteomics.2013).

[48] G.J. Stout, E.C. Stigter, P.B. Essers, K.W. Mulder, A. Kolkman, D.S. Snijders, N.J. van den Broek, M.C. Betist, H.C. Korswagen, A.W. Macinnes, A.B. Brenkman, Insulin/IGF-1-mediated longevity is marked by reduced protein metabolism. Mol.Syst.Biol. 9 (2013) 679.

[49] D. Knoefler, M. Thamsen, M. Koniczek, N.J. Niemuth, A.K. Diederich, U. Jakob, Quantitative in vivo redox sensors uncover oxidative stress as an early event in life. Mol.Cell 47 (2012) 767-776.

[50] P. Reis-Rodrigues, G. Czerwieniec, T.W. Peters, U.S. Evani, S. Alavez, E.A. Gaman, M. Vantipalli, S.D. Mooney, B.W. Gibson, G.J. Lithgow, R.E. Hughes, Proteomic analysis of age-dependent changes in protein solubility identifies genes that modulate lifespan. Aging Cell 11 (2012) 120-127.

[51] A. Canuelo, J. Peragon, Proteomics analysis in Caenorhabditis elegans to elucidate the response induced by tyrosol, an olive phenol that stimulates longevity and stress resistance. Proteomics.2013). 
[52] S. Kandasamy, D. Fan, J.S. Sangha, W. Khan, F. Evans, A.T. Critchley, B. Prithiviraj, Tasco $((\mathrm{R}))$, a product of Ascophyllum nodosum, imparts thermal stress tolerance in Caenorhabditis elegans. Mar.Drugs 9 (2011) 2256-2282.

[53] W. De Haes, L. Frooninckx, A.R. Van, A. Smolders, G. Depuydt, J. Billen, B.P. Braeckman, L. Schoofs, L. Temmerman, Metformin promotes lifespan through mitohormesis via the peroxiredoxin PRDX-2. Proc.Natl.Acad.Sci.U.S.A 111 (2014) E2501-E2509.

[54] D. Ortuno-Sahagun, M. Pallas, A.E. Rojas-Mayorquin, Oxidative stress in aging: advances in proteomic approaches. Oxid.Med.Cell Longev. 2014 (2014) 573208.

[55] A. Bogaerts, L. Temmerman, B. Boerjan, S.J. Husson, L. Schoofs, P. Verleyen, A differential proteomics study of Caenorhabditis elegans infected with Aeromonas hydrophila. Dev.Comp Immunol. 34 (2010) 690-698.

[56] A. Bogaerts, I. Beets, L. Temmerman, L. Schoofs, P. Verleyen, Proteome changes of Caenorhabditis elegans upon a Staphylococcus aureus infection. Biol.Direct. 5 (2010) 11.

[57] S. Durai, N. Singh, S. Kundu, K. Balamurugan, Proteomic investigation of Vibrio alginolyticus challenged Caenorhabditis elegans revealed regulation of cellular homeostasis proteins and their role in supporting innate immune system. Proteomics 14 (2014) 1820-1832.

[58] K.T. Simonsen, J. Moller-Jensen, A.R. Kristensen, J.S. Andersen, D.L. Riddle, B.H. Kallipolitis, Quantitative proteomics identifies ferritin in the innate immune response of C. elegans. Virulence. 2 (2011) 120-130.

[59] C. Couillault, P. Fourquet, M. Pophillat, J.J. Ewbank, A UPR-independent infectionspecific role for a BiP/GRP78 protein in the control of antimicrobial peptide expression in C. elegans epidermis. Virulence. 3 (2012) 299-308.

[60] W. Yang, K. Dierking, D. Esser, A. Tholey, M. Leippe, P. Rosenstiel, H. Schulenburg, Overlapping and unique signatures in the proteomic and transcriptomic responses of the nematode Caenorhabditis elegans toward pathogenic Bacillus thuringiensis. Dev.Comp Immunol. 51 (2015) 1-9.

[61] C. Treitz, L. Cassidy, A. Hockendorf, M. Leippe, A. Tholey, Quantitative proteome analysis of Caenorhabditis elegans upon exposure to nematicidal Bacillus thuringiensis. J.Proteomics 113 (2015) 337-350.

[62] K.T. Simonsen, S.F. Gallego, N.J. Faergeman, B.H. Kallipolitis, Strength in numbers: "Omics" studies of C. elegans innate immunity. Virulence. 3 (2012) 477-484.

[63] X. Fan, Y.M. She, R.D. Bagshaw, J.W. Callahan, H. Schachter, D.J. Mahuran, Identification of the hydrophobic glycoproteins of Caenorhabditis elegans. Glycobiol. 15 (2005) 952-964.

[64] H. Kaji, H. Saito, Y. Yamauchi, T. Shinkawa, M. Taoka, J. Hirabayashi, K. Kasai, N. Takahashi, T. Isobe, Lectin affinity capture, isotope-coded tagging and mass spectrometry to identify N-linked glycoproteins. Nat.Biotechnol. 21 (2003) 667-672. 
[65] J. Lee, K.Y. Kim, H.J. Joo, H. Kim, P.Y. Jeong, Y.K. Paik, Methods for evaluating the Caenorhabditis elegans dauer state: standard dauer-formation assay using synthetic daumones and proteomic analysis of O-GlcNAc modifications. Methods Cell Biol. 106 (2011) 445-460.

[66] D.F. Zielinska, F. Gnad, M. Jedrusik-Bode, J.R. Wisniewski, M. Mann, Caenorhabditis elegans has a phosphoproteome atypical for metazoans that is enriched in developmental and sex determination proteins. J.Proteome.Res. 8 (2009) 4039-4049.

[67] S.J. Husson, E. Clynen, G. Baggerman, A. De Loof, L. Schoofs, Discovering neuropeptides in Caenorhabditis elegans by two dimensional liquid chromatography and mass spectrometry. Biochem.Biophys.Res.Commun. 335 (2005) 76-86.

[68] S.J. Husson, B. Landuyt, T. Nys, G. Baggerman, K. Boonen, E. Clynen, M. Lindemans, T. Janssen, L. Schoofs, Comparative peptidomics of Caenorhabditis elegans versus $C$. briggsae by LC - MALDI-TOF MS. Peptides 30 (2009) 449-457.

[69] J.L. Jarecki, B.L. Frey, L.M. Smith, A.O. Stretton, Discovery of neuropeptides in the nematode Ascaris suum by database mining and tandem mass spectrometry. J.Proteome.Res. 10 (2011) 3098-3106.

[70] S.J. Husson, A. Reumer, L. Temmerman, W. De Haes, L. Schoofs, I. Mertens, G. Baggerman, Worm peptidomics. EuPA Open Proteomics 3 (2014) 280-290.

[71] S.J. Husson, E. Clynen, G. Baggerman, T. Janssen, L. Schoofs, Defective processing of neuropeptide precursors in Caenorhabditis elegans lacking proprotein convertase 2 (KPC-2/EGL-3): mutant analysis by mass spectrometry. J.Neurochem. 98 (2006) 19992012.

[72] S.J. Husson, L. Schoofs, Altered neuropeptide profile of Caenorhabditis elegans lacking the chaperone protein 7B2 as analyzed by mass spectrometry. FEBS Lett. 581 (2007) 4288-4292.

[73] S.J. Husson, T. Janssen, G. Baggerman, B. Bogert, A.H. Kahn-Kirby, K. Ashrafi, L. Schoofs, Impaired processing of FLP and NLP peptides in carboxypeptidase E (EGL21)-deficient Caenorhabditis elegans as analysed by mass spectrometry. J.Neurochem. 102 (2007) 246-260.

[74] S. Li, C.M. Armstrong, N. Bertin, H. Ge, S. Milstein, M. Boxem, P.O. Vidalain, J.D. Han, A. Chesneau, T. Hao, D.S. Goldberg, N. Li, M. Martinez, J.F. Rual, P. Lamesch, L. Xu, M. Tewari, S.L. Wong, L.V. Zhang, G.F. Berriz, L. Jacotot, P. Vaglio, J. Reboul, T. Hirozane-Kishikawa, Q. Li, H.W. Gabel, A. Elewa, B. Baumgartner, D.J. Rose, H. Yu, S. Bosak, R. Sequerra, A. Fraser, S.E. Mango, W.M. Saxton, S. Strome, S. Van Den Heuvel, F. Piano, J. Vandenhaute, C. Sardet, M. Gerstein, L. Doucette-Stamm, K.C. Gunsalus, J.W. Harper, M.E. Cusick, F.P. Roth, D.E. Hill, M. Vidal, A map of the interactome network of the metazoan C. elegans. Science 303 (2004) 540-543.

[75] N. Simonis, J.F. Rual, A.R. Carvunis, M. Tasan, I. Lemmens, T. Hirozane-Kishikawa, T. Hao, J.M. Sahalie, K. Venkatesan, F. Gebreab, S. Cevik, N. Klitgord, C. Fan, P. Braun, N. Li, N. Ayivi-Guedehoussou, E. Dann, N. Bertin, D. Szeto, A. Dricot, M.A. Yildirim, C. Lin, A.S. de Smet, H.L. Kao, C. Simon, A. Smolyar, J.S. Ahn, M. Tewari, M. Boxem, S. Milstein, H. Yu, M. Dreze, J. Vandenhaute, K.C. Gunsalus, M.E. Cusick, 
D.E. Hill, J. Tavernier, F.P. Roth, M. Vidal, Empirically controlled mapping of the Caenorhabditis elegans protein-protein interactome network. Nat.Methods 6 (2009) 4754.

[76] W. Zhong, P.W. Sternberg, Genome-wide prediction of C. elegans genetic interactions. Science 311 (2006) 1481-1484.

[77] L. Stein, P. Sternberg, R. Durbin, J. Thierry-Mieg, J. Spieth, WormBase: network access to the genome and biology of Caenorhabditis elegans. Nucleic Acids Res. 29 (2001) 8286. 